\title{
Bell nonlocality in the turbulent atmosphere
}

\author{
M.O. Gumberidze, ${ }^{1,2,3}$ A.A. Semenov, ${ }^{1,4}$ D. Vasylyev, ${ }^{4,5}$ and W. Vogel $^{4}$ \\ ${ }^{1}$ Institute of Physics, National Academy of Sciences of Ukraine, Prospect Nauky 46, 03028 Kiev, Ukraine \\ ${ }^{2}$ Physics Department, Taras Shevchenko National University of Kiev, Prospect Glushkova 2, 03022 Kiev, Ukraine \\ ${ }^{3}$ Department of Optics, Palacký University, 17. listopadu 12, 77146 Olomouc, Czech Republic \\ ${ }^{4}$ Institut für Physik, Universität Rostock, Albert-Einstein-Straße 23, D-18059 Rostock, Germany \\ ${ }^{5}$ Bogolyubov Institute for Theoretical Physics, NAS of Ukraine, \\ Vulytsya Metrologichna 14-b, 03680 Kiev, Ukraine
}

\begin{abstract}
Violations of Bell inequalities are better preserved by turbulent atmospheric channels than by comparable optical fibers in the scenario of copropagating entangled photons [A. A. Semenov and W. Vogel, Phys. Rev. A 81, 023835 (2010); arXiv:0909.2492. Here we reexamine this result for the case of counterpropagation also considering the fact that each receiver registers so-called doubleclick events, which are caused by dark counts, stray light, and multi-photon entangled pairs. We show that advantages of the atmospheric links are feasible only for the copropagation scenario in the case of strong fluctuations of losses. For counterpropagation, the violations of Bell inequalities can be improved with an additional postselection procedure testing the channel transmittance.
\end{abstract}

PACS numbers: 03.65.Ud, 42.68.Ay, 42.65.Lm

\section{INTRODUCTION}

The interest in quantum key distribution (QKD) schemes [1] through free-space channels is due to the intriguing practical perspectives of information security in the scenarios of communication between mobile participants, links through hardly accessible regions, global quantum communications via satellites [2 7], etc. QKD protocols using Bell inequalities (for a review see Ref. [8] ) assume sharing of radiation-field modes between remote participants. Nonclassical fields may violate the Bell inequalities. This corresponds to the absence of locality and/or realism (local realism) in quantum physics. We refer to this phenomenon, in accordance with [8, as Bell nonlocality. In this context, the original Ekert protocol (E91) 9] utilizes the Bell inequalities to test for eavesdropping. Moreover, the relevance of Bell inequalities has been recognized in the context of device-independent QKD (cf. Refs. 10, 11). The corresponding protocols do not depend on detailed characterizations of measurement devices, and eavesdroppers may even have some control of them.

Experimental violations of Bell inequalities for light passing through the atmosphere have been demonstrated for a $144 \mathrm{~km}$ channel on the Canary Islands [12, 13. A consistent theoretical analysis of such types of experiments requires a deep understanding of destructive phenomena. This includes generation, transmission, and detection of nonclassical light. Fading effects, i.e., fluctuating losses (cf. Refs [14, 15]), absorption, and noise events originating from dark counts or stray light are serious obstacles for proper tests of the Bell inequalities.

In a Bell-like experiment implemented in a $144 \mathrm{~km}$ atmospheric channel [13, the scenario of copropagation has been studied. In that case two photons, which were prepared at the parametric down-conversion (PDC) source in a polarization-entangled state, were sent in the same direction from the transmitter to the receiver with a tem- poral separation much smaller than the characteristic time scales of atmospheric variations (see Refs. 16, 17] for other types of entangled states prepared by the PDC source, which are useful for atmospheric communications). Such fading channels can be considered to have correlated transmittances. For this scenario the turbulence may even improve measured values of the Bell parameter [18. This effect can be easily explained by the fact that correlated counts from the source are more likely to be detected when the channel is randomly transparent. On the other hand, for events with low transmittances the detection of simultaneous noise clicks occurs with lower probabilities.

Multi-photon pairs from PDC sources [19, 20] and noise counts also lead to the appearance of so-called double-click events, which make it impossible to ascribe a definite value of the qubit for the corresponding measurements 21. The exclusion of such events from consideration may lead to a lower security of QKD protocols. Instead, one assigns a random value to the corresponding qubit. This technique, usually referred to as the squash model 22 24, enables one to perform a consistent mapping of continuous-variable PDC states onto discrete-variable qubit states.

In the present paper we consider violations of Bell inequalities caused by light being in a polarizationentangled state, which is transmitted through the atmosphere, and address two main issues. First, we study how the incorporation of double-click events in the framework of the corresponding squash models influences the feasibility of checking the Bell-inequality violation in turbulent-atmosphere channels. Second, we demonstrate that for the counterpropagation scenario the advantages of atmospheric channels are not directly feasible. However, the advantages can still be utilized by applying a postselection procedure testing the channel transmittance.

The paper is organized as follows. In Sec. II we derive 
relations for the correlation coefficients and Bell parameters in the presence of double-click events and for scenarios of copropagating and counterpropagating fields. In Sec. III we analyze the effect of double-click events on the Bell-inequality violation in the case of copropagation. The case of counterpropagation is considered in Sec. IV. In Sec. V V we consider a postselection procedure, which enables us to improve the violations of Bell inequalities. In Sec. VI we summarize our results and give some conclusions.

\section{BELL INEQUALITIES FOR ATMOSPHERIC CHANNELS}

\section{A. Design of the experiment}

Let us recall the physical background of Bell-inequality tests in atmospheric channels [12, 13, 18. We distinguish between two scenarios: the first one with different modes of the entangled light copropagating in the same direction and the second one with the modes being counterpropagating (cf. Fig. 1). The PDC source generates entangled photons, which are sent through the turbulent atmosphere to receivers $\mathrm{A}$ and $\mathrm{B}$. In the copropagation scenario one photon is sent directly and the other one through a delay line to the corresponding receiver. There the photons are analyzed with polarization analyzers. Each of them consists of a half-wave plate (HWP), rotating the polarization by the angles $\theta_{\mathrm{A}}$ and $\theta_{\mathrm{B}}$, polarizing beam splitters (PBS), and click detectors for the transmission and reflection channels, $D_{T_{\mathrm{A}(\mathrm{B})}}$ and $\mathrm{D}_{R_{\mathrm{A}(\mathrm{B})}}$, respectively. The scheme of copropagation additionally includes a 50:50 beam splitter (BS), which randomly selects photons propagating to receivers $\mathrm{A}$ and $\mathrm{B}$ with the unavoidable introduction of $3-\mathrm{dB}$ deterministic losses (cf. Ref. 13). In the scenario of counterpropagation, the entangled photons are sent in different directions through different atmospheric channels. The photons reach the receivers $\mathrm{A}$ and $\mathrm{B}$, where they are analyzed with the corresponding polarization analyzers.

If the detector $\mathrm{D}_{T_{\mathrm{A}(\mathrm{B})}}$ in the corresponding transmission channel clicks, this means that the incoming photon is linearly polarized with the angle $\theta_{\mathrm{A}(\mathrm{B})}$. If the detector $\mathrm{D}_{R_{\mathrm{A}(\mathrm{B})}}$ in the corresponding reflection channel clicks, the incoming photon is assumed to be polarized in the orthogonal direction. For our purposes it is important to count the simultaneous clicks on the sites $\mathrm{A}$ and $\mathrm{B}$ in order to reconstruct the frequencies $P_{i_{\mathrm{A}}, i_{\mathrm{B}}}\left(\theta_{\mathrm{A}}, \theta_{\mathrm{B}}\right)$, where $i_{\mathrm{A}(\mathrm{B})}=\left\{T_{\mathrm{A}(\mathrm{B})}, R_{\mathrm{A}(\mathrm{B})}\right\}$. The frequency

$$
P_{\text {same }}\left(\theta_{\mathrm{A}}, \theta_{\mathrm{B}}\right)=P_{T_{\mathrm{A}}, T_{\mathrm{B}}}\left(\theta_{\mathrm{A}}, \theta_{\mathrm{B}}\right)+P_{R_{\mathrm{A}}, R_{\mathrm{B}}}\left(\theta_{\mathrm{A}}, \theta_{\mathrm{B}}\right),
$$

of simultaneous clicks of the pair of detectors $D_{T_{\mathrm{A}}}$ and $\mathrm{D}_{T_{\mathrm{B}}}$ or the pair $\mathrm{D}_{R_{\mathrm{A}}}$ and $\mathrm{D}_{R_{\mathrm{B}}}$, and the frequency,

$$
P_{\text {different }}\left(\theta_{\mathrm{A}}, \theta_{\mathrm{B}}\right)=P_{T_{\mathrm{A}}, R_{\mathrm{B}}}\left(\theta_{\mathrm{A}}, \theta_{\mathrm{B}}\right)+P_{R_{\mathrm{A}}, T_{\mathrm{B}}}\left(\theta_{\mathrm{A}}, \theta_{\mathrm{B}}\right) \text {, }
$$

of simultaneous clicks of the pair of detectors $D_{T_{\mathrm{A}}}$ and $\mathrm{D}_{R_{\mathrm{B}}}$ or $\mathrm{D}_{R_{\mathrm{A}}}$ and $\mathrm{D}_{T_{\mathrm{B}}}$ is then used for evaluation of the (a)

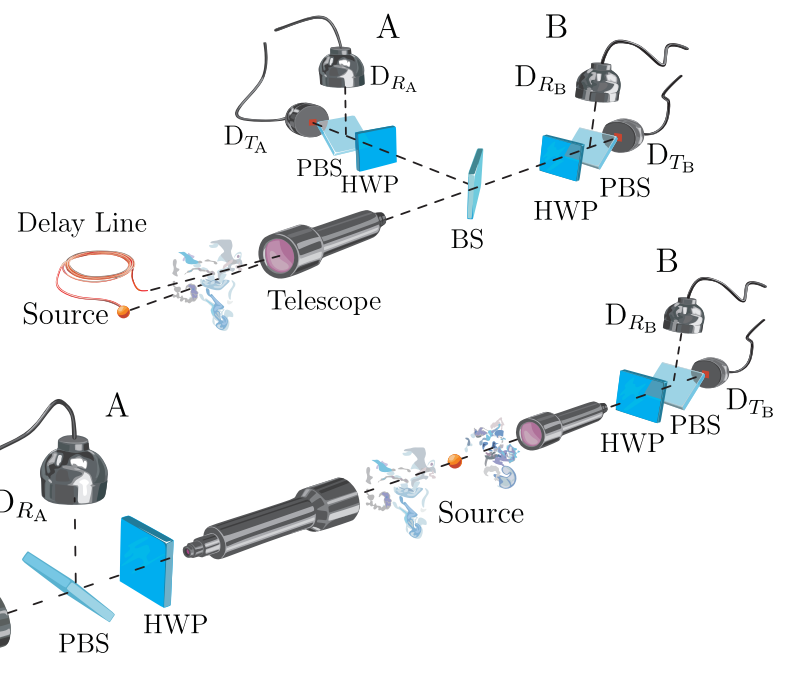

FIG. 1. (Color online) Typical experimental setups for verification of Bell inequalities in atmospheric channels: (a) the copropagation scheme as used in Ref. 13; (b) the counterpropagation scheme. For more details, see the text.

correlation coefficients

$$
E\left(\theta_{\mathrm{A}}, \theta_{\mathrm{B}}\right)=\frac{P_{\text {same }}\left(\theta_{\mathrm{A}}, \theta_{\mathrm{B}}\right)-P_{\text {different }}\left(\theta_{\mathrm{A}}, \theta_{\mathrm{B}}\right)}{P_{\text {same }}\left(\theta_{\mathrm{A}}, \theta_{\mathrm{B}}\right)+P_{\text {different }}\left(\theta_{\mathrm{A}}, \theta_{\mathrm{B}}\right)} .
$$

The Bell theorem in the Clauser-Horne-Shimony-Holt (CHSH) form [8, 25] states that for two sets of polarization angles the Bell parameter

$$
\begin{aligned}
\mathcal{B} & =\left|E\left(\theta_{\mathrm{A}}^{(1)}, \theta_{\mathrm{B}}^{(1)}\right)-E\left(\theta_{\mathrm{A}}^{(1)}, \theta_{\mathrm{B}}^{(2)}\right)\right| \\
& +\left|E\left(\theta_{\mathrm{A}}^{(2)}, \theta_{\mathrm{B}}^{(2)}\right)+E\left(\theta_{\mathrm{A}}^{(2)}, \theta_{\mathrm{B}}^{(1)}\right)\right|,
\end{aligned}
$$

satisfies the condition

$$
\mathcal{B} \leq 2
$$

for local realistic theories. For nonclassical light fields the Bell inequality can be violated. For such nonclassical fields the correlation properties are incompatible with those of any local model. Violation of inequality (5) certifies the presence of nonlocal quantum correlations of light.

\section{B. Theoretical analysis}

An analysis of the Bell-inequality test requires derivation of the joint probabilities, $P_{i_{\mathrm{A}}, i_{\mathrm{B}}}\left(\theta_{\mathrm{A}}, \theta_{\mathrm{B}}\right)$, for detection of photons at receiver sites $\mathrm{A}$ and $\mathrm{B}$. The derivation of these quantities can be performed on a similar footing as done in Ref. 18. However, here we should not omit double clicks, i.e., events where detectors in transmission and reflection channels click simultaneously at at least one site, A or B. This may happen due to the stray light, dark counts and multi-photon pairs. According to 
the photodetection theory [26, 27] we can write the joint probabilities of photon detection as

$$
\begin{aligned}
P_{i_{\mathrm{A}}, i_{\mathrm{B}}}\left(\theta_{\mathrm{A}}, \theta_{\mathrm{B}}\right) & =\operatorname{Tr}\left(\hat{\Pi}_{i_{\mathrm{A}}}^{(c)} \hat{\Pi}_{i_{\mathrm{B}}}^{(c)} \hat{\Pi}_{j_{\mathrm{A}}}^{(0)} \hat{\Pi}_{j_{\mathrm{B}}}^{(0)} \hat{\rho}\right) \\
& +\frac{1}{2} \operatorname{Tr}\left(\hat{\Pi}_{i_{\mathrm{A}}}^{(c)} \hat{\Pi}_{i_{\mathrm{B}}}^{(c)} \hat{\Pi}_{j_{\mathrm{A}}}^{(c)} \hat{\Pi}_{j_{\mathrm{B}}}^{(0)} \hat{\rho}\right) \\
& +\frac{1}{2} \operatorname{Tr}\left(\hat{\Pi}_{i_{\mathrm{A}}}^{(c)} \hat{\Pi}_{i_{\mathrm{B}}}^{(c)} \hat{\Pi}_{j_{\mathrm{A}}}^{(0)} \hat{\Pi}_{j_{\mathrm{B}}}^{(c)} \hat{\rho}\right) \\
& +\frac{1}{4} \operatorname{Tr}\left(\hat{\Pi}_{i_{\mathrm{A}}}^{(c)} \hat{\Pi}_{i_{\mathrm{B}}}^{(c)} \hat{\Pi}_{j_{\mathrm{A}}}^{(c)} \hat{\Pi}_{j_{\mathrm{B}}}^{(c)} \hat{\rho}\right),
\end{aligned}
$$

where $\hat{\rho}$ is the density operator and

$$
\begin{aligned}
& \hat{\Pi}_{i_{\mathrm{A}(\mathrm{B})}}^{(0)}=: \exp \left(-\eta_{c} \hat{a}_{i_{\mathrm{A}(\mathrm{B})}^{\dagger}}^{\dagger} \hat{a}_{i_{\mathrm{A}(\mathrm{B})}}-\nu\right):, \\
& \hat{\Pi}_{i_{\mathrm{A}(\mathrm{B})}^{(c)}}^{(c)}=1-: \exp \left(-\eta_{c} \hat{a}_{i_{\mathrm{A}(\mathrm{B})}^{\dagger}}^{\dagger} \hat{a}_{i_{\mathrm{A}(\mathrm{B})}}-\nu\right):
\end{aligned}
$$

are the positive operator-valued measures for the on/off detectors $i_{\mathrm{A}(\mathrm{B})}$, related to the absence and presence of detection events, respectively. Here $\hat{a}_{i_{\mathrm{A}(\mathrm{B})}}$ and $\hat{a}_{i_{\mathrm{A}(\mathrm{B})}^{\dagger}}^{\dagger}$ are photonic annihilation and creation operators, respectively, for the field modes at the $i_{\mathrm{A}(\mathrm{B})}=\left\{T_{\mathrm{A}(\mathrm{B})}, R_{\mathrm{A}(\mathrm{B})}\right\}$ output of the PBS such that $i_{\mathrm{A}(\mathrm{B})} \neq j_{\mathrm{A}(\mathrm{B})}, \eta_{c}$ is the detection efficiency, $\nu$ is the mean number of stray-light and dark counts [28], and : ... : denotes the normal ordering.

The last three terms in Eq. (6) describe the contributions from double-click events, when both detectors on at least one side click. For such events we assign random values of the corresponding qubits. In practice this means that if both detectors, $\mathrm{D}_{T_{\mathrm{A}(\mathrm{B})}}$ and $\mathrm{D}_{R_{\mathrm{A}(\mathrm{B})}}$, at site $\mathrm{A}$ (B) click simultaneously, we randomly ascribe to such a realization the event related to the click on detector $\mathrm{D}_{T_{\mathrm{A}(\mathrm{B})}}$ or $\mathrm{D}_{R_{\mathrm{A}(\mathrm{B})}}$ with probability $1 / 2$. In the case where all four detectors click simultaneously we randomly ascribe to this realization any of four possible pair events with probability $1 / 4$. The three terms associated with double-click events have not been considered in Ref. [18. In the present article we analyze the Bell inequality taking these contributions into account.

We now specify the quantum state, $\hat{\rho}=|\mathrm{PDC}\rangle\langle\mathrm{PDC}|$, generated by the PDC source (cf. Refs. [19, 20]):

$$
|\mathrm{PDC}\rangle=(\cosh \xi)^{-2} \sum_{n=0}^{+\infty} \sqrt{n+1} \tanh ^{n} \xi\left|\Phi_{n}\right\rangle .
$$

Herein $\xi$ is the squeezing parameter,

$$
\begin{aligned}
& \left|\Phi_{n}\right\rangle= \\
& \frac{1}{\sqrt{n+1}} \sum_{m=0}^{n}(-1)^{m}|n-m\rangle_{\mathrm{H}_{\mathrm{A}}}|m\rangle_{\mathrm{V}_{\mathrm{A}}}|m\rangle_{\mathrm{H}_{\mathrm{B}}}|n-m\rangle_{\mathrm{V}_{\mathrm{B}}},
\end{aligned}
$$

and $|n\rangle_{\mathrm{H}_{\mathrm{A}(\mathrm{B})}}$ and $|n\rangle_{\mathrm{V}_{\mathrm{A}(\mathrm{B})}}$ are the photon-number states of the horizontal and vertical polarization modes, respectively, sent to the receiver $\mathrm{A}(\mathrm{B})$. For small values of $\xi$ the first two terms in Eq. (9) are the most relevant, such that $\left|\Phi_{0}\right\rangle$ is the vacuum state and

$$
\begin{aligned}
& \left|\Phi_{1}\right\rangle= \\
& \frac{1}{\sqrt{2}}\left(|1\rangle_{\mathrm{H}_{\mathrm{A}}}|0\rangle_{\mathrm{V}_{\mathrm{A}}}|0\rangle_{\mathrm{H}_{\mathrm{B}}}|1\rangle_{\mathrm{V}_{\mathrm{B}}}-|0\rangle_{\mathrm{H}_{\mathrm{A}}}|1\rangle_{\mathrm{V}_{\mathrm{A}}}|1\rangle_{\mathrm{H}_{\mathrm{B}}}|0\rangle_{\mathrm{V}_{\mathrm{B}}}\right)
\end{aligned}
$$

is the Bell state,

$$
|\mathcal{B}\rangle \equiv\left|\Phi_{1}\right\rangle=\frac{1}{\sqrt{2}}\left(|\mathrm{H}\rangle_{\mathrm{A}}|\mathrm{V}\rangle_{\mathrm{B}}-|\mathrm{V}\rangle_{\mathrm{A}}|\mathrm{H}\rangle_{\mathrm{B}}\right)
$$

which maximally violates inequality (5). Here the state $|\mathrm{H}\rangle_{\mathrm{A}(\mathrm{B})}=|1\rangle_{\mathrm{H}_{\mathrm{A}(\mathrm{B})}}|0\rangle_{\mathrm{V}_{\mathrm{A}(\mathrm{B})}}$ corresponds to the photon in the horizontal mode at site $\mathrm{A}(\mathrm{B})$, whereas the state $|\mathrm{V}\rangle_{\mathrm{A}(\mathrm{B})}=|0\rangle_{\mathrm{H}_{\mathrm{A}(\mathrm{B})}}|1\rangle_{\mathrm{V}_{\mathrm{A}(\mathrm{B})}}$ corresponds to the photon in the vertical mode at site $A(B)$.

Further analysis is performed similarly to that in Ref. [18; for details of calculations see also Appendixes C and D. First, we should modify the initial quantum state [cf. Eqs. (9) or [11)] according to the quantum-state input-output relation for fading channels (cf. Ref. [14, 15]) with fluctuating transmission efficiencies (transmittances) $\eta_{\mathrm{A}}$ for modes $\mathrm{H}_{A} \mathrm{~V}_{A}$ and $\eta_{\mathrm{B}}$ for modes $\mathrm{H}_{B} \mathrm{~V}_{B}$. This relation has a simple form in the Glauber-Sudarshan $P$ representation,

$$
P_{\text {out }}(\boldsymbol{\alpha})=\int_{0}^{1} \mathrm{~d}^{2} \boldsymbol{\eta} \frac{1}{\eta_{\mathrm{A}}^{2} \eta_{\mathrm{B}}^{2}} \mathcal{P}\left(\eta_{\mathrm{A}}, \eta_{\mathrm{B}}\right) P_{\text {in }}\left(\boldsymbol{\alpha}_{\boldsymbol{\eta}}\right),
$$

where $P_{\text {in }}$ and $P_{\text {out }}$ are the Glauber-Sudarshan $P$ functions (cf. Ref. 29 31) of light at the source [corresponding to the state (9) or (11)] and at the receivers, respectively, $\boldsymbol{\alpha}=\left(\alpha_{H_{\mathrm{A}}}, \alpha_{V_{\mathrm{A}}}, \alpha_{H_{\mathrm{B}}}, \alpha_{V_{\mathrm{B}}}\right)$ and $\boldsymbol{\alpha}_{\boldsymbol{\eta}}=\left(\alpha_{H_{\mathrm{A}}} / \sqrt{\eta}_{\mathrm{A}}, \alpha_{V_{\mathrm{A}}} / \sqrt{\eta}_{\mathrm{A}}, \alpha_{H_{\mathrm{B}}} / \sqrt{\eta}_{\mathrm{B}}, \alpha_{V_{\mathrm{B}}} / \sqrt{\eta}_{\mathrm{B}}\right)$, $\mathrm{d}^{2} \boldsymbol{\eta}=\mathrm{d} \eta_{\mathrm{A}} \mathrm{d} \eta_{\mathrm{B}}$. In this equation the probability distribution of the transmittance $(\mathrm{PDT}), \mathcal{P}\left(\eta_{\mathrm{A}}, \eta_{\mathrm{B}}\right)$, is the main characteristics of the atmospheric channels.

The explicit form of the PDT depends on the characteristics of the irradiated light beam, radius of the receiver aperture, and characteristics of the atmospheric channel, i.e., its length and turbulence conditions. For characterization of channels with homogeneous and isotropic turbulence we use the Rytov parameter (cf. Refs. 32 35]),

$$
\sigma_{R}^{2}=1.23 C_{n}^{2} k^{\frac{7}{6}} L^{\frac{11}{6}}
$$

which quantifies the integral effect of optical turbulence on the whole channel. Here $C_{n}^{2}$ is the index-of-refraction structure constant, characterizing the local strength of turbulence, $k$ is the wave number of optical radiation, and $L$ is the channel length. Throughout this article, we consider weak- to moderate-turbulence channels $\left(\sigma_{R}^{2} \approx 1 \ldots 10\right)$ and strong-turbulence $\left(\sigma_{R}^{2} \gg 1\right)$ channels. These notions characterize the integral effects of the turbulence on the quantum light over the whole propagation paths under study. 
Derivation of the PDT applies the knowledge of classical atmospheric optics (see e.g. [32 39]). For weakturbulence channels, $\sigma_{R}<1$, when the leading disturbance is beam wandering, the PDT takes the form of the lognegative Weibull distribution (see Ref. 15 for its derivation). For weak- to moderate- and strong-turbulence channels, deformations of the beam play an important role. The corresponding PDT in the elliptic-beam approximation has been derived in Ref. 40. It is important to note that under strong-turbulence conditions the elliptic-beam model is in reasonable agreement with the log-normal distribution [41,44, provided that the latter is restricted to the physical domain, $\eta_{\mathrm{A}(\mathrm{B})} \leq 1$. Such behavior has been experimentally verified in Ref. 45]. In the present paper we apply two scenarios with the corresponding PDTs. First, we consider a $1.6 \mathrm{~km}$ channel in the city of Erlangen (cf. Ref. [46]) as a typical example of weak- to moderate-turbulence channels. The corresponding experimental results are in good agreement with the recently proposed elliptic-beam model for the PDT (see Ref. [40] and Appendix A for more details). Second, we consider a $144-\mathrm{km}$ channel on the Canary Islands (cf. 45]) as a typical strong-turbulence channel. For simplicity, the corresponding PDT is approximated in the following by the truncated log-normal distribution (for more details see Appendix B).

For the purposes of this paper it is important to note that within the given apertures the weak- to moderateturbulence channel is characterized by a small value of the fluctuation of losses, $\left\langle\Delta \eta^{2}\right\rangle /\langle\eta\rangle^{2}$. The strongturbulence channel has a large value of this parameter. It is noteworthy that higher moments of the transmittance may also play a crucial role for characterization of the considered channels.

As the next step, we rewrite the state in terms of transmitted, $T_{\mathrm{A}(B)}$, and reflected, $R_{\mathrm{A}(B)}$, modes in the inputs of polarization-analyzer detectors. The corresponding input-output relations for the field operators read as

$$
\begin{aligned}
& \hat{a}_{\mathrm{H}_{\mathrm{A}(\mathrm{B})}}=\hat{a}_{T_{\mathrm{A}(\mathrm{B})}} \cos \theta_{\mathrm{A}(\mathrm{B})}-\hat{a}_{R_{\mathrm{A}(\mathrm{B})}} \sin \theta_{\mathrm{A}(\mathrm{B})}, \\
& \hat{a}_{\mathrm{V}_{\mathrm{A}(\mathrm{B})}}=\hat{a}_{T_{\mathrm{A}(\mathrm{B})}} \sin \theta_{\mathrm{A}(\mathrm{B})}+\hat{a}_{R_{\mathrm{A}(\mathrm{B})}} \cos \theta_{\mathrm{A}(\mathrm{B})},
\end{aligned}
$$

where $\hat{a}_{\mathrm{H}_{\mathrm{A}(\mathrm{B})}}$ and $\hat{a}_{\mathrm{V}_{\mathrm{A}(\mathrm{B})}}$ are field annihilation operators for the site $\mathrm{A}(\mathrm{B})$ horizontal and vertical modes, respectively. Technical details of applications of these relations can be found in Ref. [18] as well as in Appendixes C and D. The obtained density operator is substituted in Eq. (6) and then in Eqs. (1) and (2), which leads to explicit forms of $P_{\text {same }}\left(\theta_{\mathrm{A}}, \theta_{\mathrm{B}}\right)$ and $P_{\text {different }}\left(\theta_{\mathrm{A}}, \theta_{\mathrm{B}}\right)$. Finally, we use these probabilities for calculations of the correlation coefficients, (3), and maximization of the Bell parameter, (4), with respect to the angles $\theta_{\mathrm{A}}$ and $\theta_{\mathrm{B}}$.

\section{Parametric down-conversion source}

In the case of the PDC source the initial state is given by the density operator $\hat{\rho}=|\mathrm{PDC}\rangle\langle\mathrm{PDC}|[\mathrm{cf}$. Eq. (9)]. Applying the above discussed analysis (cf. also Appendix C), one gets for the probabilities $P_{\text {same }}\left(\theta_{\mathrm{A}}, \theta_{\mathrm{B}}\right)$ and $P_{\text {different }}\left(\theta_{\mathrm{A}}, \theta_{\mathrm{B}}\right)$

$$
\begin{aligned}
& P_{\mathrm{i}}\left(\theta_{\mathrm{A}}, \theta_{\mathrm{B}}\right)=\frac{1}{2}+\frac{e^{-4 \nu}}{2}\left(1-\tanh ^{2} \xi\right)^{4} \\
& \times\left[e^{2 \nu}\left(2\left\langle\frac{1}{C_{0}+C_{1 \mathrm{~A}}+C_{1 \mathrm{~B}}+C_{\mathrm{i}}}\right\rangle-\left\langle\frac{C_{0}}{\left(C_{0}+C_{1 \mathrm{~A}}\right)^{2}}\right\rangle-\left\langle\frac{C_{0}}{\left(C_{0}+C_{1 \mathrm{~B}}\right)^{2}}\right\rangle-2\left\langle\frac{1}{C_{0}+C_{1 \mathrm{~A}}+C_{1 \mathrm{~B}}+C_{\mathrm{j}}}\right\rangle\right)+\left\langle\frac{1}{C_{0}}\right\rangle\right]
\end{aligned}
$$

Here $i, j=\{$ same, different $\}, i \neq j$,

$$
\begin{gathered}
C_{0}=\left\{\eta_{c}^{2} \eta_{\mathrm{A}} \eta_{\mathrm{B}} \tanh ^{2} \xi-\left[1+\left(\eta_{c} \eta_{\mathrm{A}}-1\right) \tanh ^{2} \xi\right]\left[1+\left(\eta_{c} \eta_{\mathrm{B}}-1\right) \tanh ^{2} \xi\right]\right\}^{2} \\
C_{1 \mathrm{~A}(\mathrm{~B})}=\eta_{c} \eta_{\mathrm{B}(\mathrm{A})}\left(1-\eta_{c} \eta_{\mathrm{A}(\mathrm{B})}\right)\left(1-\tanh ^{2} \xi\right) \tanh ^{2} \xi \\
\times \quad\left\{\eta_{c}^{2} \eta_{\mathrm{A}(\mathrm{B})} \eta_{\mathrm{B}(\mathrm{A})} \tanh ^{2} \xi-\left[1+\left(\eta_{c} \eta_{\mathrm{A}(\mathrm{B})}-1\right) \tanh ^{2} \xi\right]\left[1+\left(\eta_{c} \eta_{\mathrm{B}(\mathrm{A})}-1\right) \tanh ^{2} \xi\right]\right\} \\
C_{\mathrm{same}}=\eta_{c}^{2} \eta_{\mathrm{A}} \eta_{\mathrm{B}} \tanh ^{2} \xi\left(1-\tanh ^{2} \xi\right)^{2}\left[\left(1-\eta_{c} \eta_{\mathrm{A}}\right)\left(1-\eta_{c} \eta_{\mathrm{B}}\right) \tanh ^{2} \xi-\sin ^{2}\left(\theta_{\mathrm{A}}-\theta_{\mathrm{B}}\right)\right] \\
C_{\text {different }}=\eta_{c}^{2} \eta_{\mathrm{A}} \eta_{\mathrm{B}} \tanh ^{2} \xi\left(1-\tanh ^{2} \xi\right)^{2}\left[\left(1-\eta_{c} \eta_{\mathrm{A}}\right)\left(1-\eta_{c} \eta_{\mathrm{B}}\right) \tanh ^{2} \xi-\cos ^{2}\left(\theta_{\mathrm{A}}-\theta_{\mathrm{B}}\right)\right]
\end{gathered}
$$

and means averaging by the channel transmittances.

$$
\langle\ldots\rangle=\int_{0}^{1} \mathrm{~d}^{2} \boldsymbol{\eta} \ldots \mathcal{P}\left(\eta_{\mathrm{A}}, \eta_{\mathrm{B}}\right)
$$




\section{Bell-state source}

For the case of a weak-intensity source, the state at the transmitter can be effectively approximated by the Bell states [cf. Eqs. (11) and (12)]. In this case calculation of the Bell parameter can be performed explicitly. The maximal value of the Bell parameter [cf. Eq. (4)] reads as

$$
\begin{aligned}
& \mathcal{B}= \\
& \frac{2 \sqrt{2} p_{\mathcal{B}} \eta_{c}^{2} e^{2 \nu}}{p_{\mathcal{B}}\left(e^{2 \nu}+\eta_{c}-1\right)^{2}+p_{0}\left(e^{2 \nu}-1\right)^{2}+p_{1}\left(e^{2 \nu}-1\right)\left(e^{2 \nu}+\eta_{c}-1\right)}
\end{aligned}
$$

Here

$$
p_{\mathcal{B}}=\left\langle\eta_{\mathrm{A}} \eta_{\mathrm{B}}\right\rangle
$$

is the probability that at the output one gets the Bell state,

$$
p_{0}=\left\langle\left(1-\eta_{\mathrm{A}}\right)\left(1-\eta_{\mathrm{B}}\right)\right\rangle
$$

is the probability that both photons do not reach the receiver, and finally

$$
p_{1}=\left\langle\eta_{\mathrm{A}}\left(1-\eta_{\mathrm{B}}\right)\right\rangle+\left\langle\eta_{\mathrm{B}}\left(1-\eta_{\mathrm{A}}\right)\right\rangle
$$

is the probability that only one photon will reach the receiver. Details of calculations can be found in Appendix D

\section{COPROPAGATION}

The scenario of copropagation, which is represented in Fig. 1(a) and was experimentally studied in [13, is characterized by a very short time interval between two entangled pulses. This interval is much less than the time, for which the atmosphere is changed. As a result, the two transmittances $\eta_{\mathrm{A}}$ and $\eta_{\mathrm{B}}$ can be considered to be completely correlated. The PDT in this case is given by

$$
\mathcal{P}\left(\eta_{\mathrm{A}}, \eta_{\mathrm{B}}\right)=\mathcal{P}\left(\eta_{\mathrm{A}}\right) \delta\left(\eta_{\mathrm{A}}-\eta_{\mathrm{B}}\right)
$$

where $\mathcal{P}\left(\eta_{\mathrm{A}}\right)$ is the single-mode PDT. This implies that Eq. 22 can be rewritten as

$$
\langle\ldots\rangle=\int_{0}^{1} \mathrm{~d} \eta \ldots \mathcal{P}(\eta),
$$

where we explicitly take that $\eta_{\mathrm{A}}=\eta=\eta_{\mathrm{B}}$.

As mentioned in Sec. I] such fading channels demonstrate higher values of the Bell parameter compared with deterministic attenuation channels characterized by the transmittance $\eta_{0}=\langle\eta\rangle$ (cf. Ref. [18]). Indeed, from the Cauchy-Schwarz inequality it follows that $\left\langle\eta^{2}\right\rangle \geq\langle\eta\rangle^{2}$. This yields that the probability of preserving Bell states by the channel [cf. Eq. (24)] is higher for the correlated fading channel compared with the deterministicloss channel, i.e.,

$$
p_{\mathcal{B}}=\left\langle\eta^{2}\right\rangle \geq \eta_{0}^{2}=p_{\mathcal{B}}^{\text {det }} .
$$

As a result, the violation of the Bell inequalities is more significant in the case of correlated atmospheric channels.

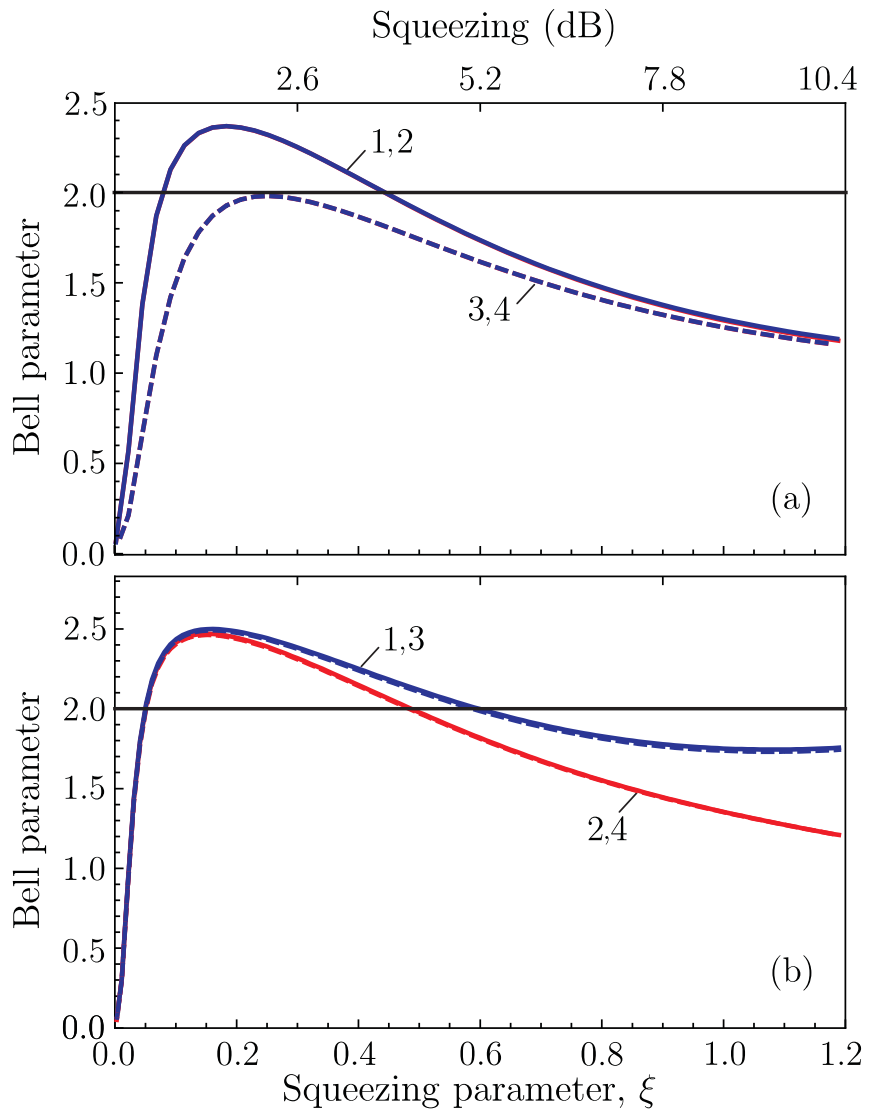

FIG. 2. (Color online) The Bell parameter, $\mathcal{B}$, vs the squeezing parameter, $\xi$, for the scenario of copropagation. The PDT is taken (a) for the strong-turbulence channel (cf. Appendix $\mathrm{B}$, where the mean number of stray-light and dark counts is $\nu=1.7 \times 10^{-5}$, and (b) for the weak- to moderateturbulence channel (cf. Appendix A), with $\nu=3 \times 10^{-3}$. For both cases, lines 1 and 2 correspond to fading channels without and with the incorporation of double-clicks events, respectively. Lines 3 and 4 correspond to the deterministic-loss channels with $\eta_{0}=\langle\eta\rangle$, without and with the incorporation of double-clicks events, respectively. The detection efficiency is $\eta_{c}=0.3$, which includes 3 - $\mathrm{dB}$ losses at the beam splitter of the receiver. Here and in the following figures, the horizontal line $\mathcal{B}=2$ shows the local-realism bound.

In Ref. [18 this property has been theoretically considered by neglecting the double-click events, i.e., Eq. (6) has been taken without the last three terms (see also Appendix E). However, the incorporation of double-click events may diminish the measured value of the Bell parameter. In the following we consider these effects.

In Fig. 2 we demonstrate the dependence of the Bell parameter, $\mathcal{B}$, on the squeezing parameter, $\xi$, of the PDC 
state [cf. Eq. (9)] for the case of incorporation of doubleclick events and for discarding it (see Ref. 18 and Appendix $\mathrm{E}$ for the latter). An interesting result is that in the case of large atmospheric losses, such as occur in the considered strong-turbulence channel (cf. Appendix B), the effect of double-click events is negligible. This can be explained by the relatively low probability of multiphoton pairs' passing through the high-loss channel even for a bright source (large squeezing parameter, $\xi$ ). A similar behavior is observed for a weak-intensity source (small squeezing parameter, $\xi$ ) in the case of weak- to moderate-turbulence channel (cf. Appendix A). In the latter case the overall losses are relatively small. As a result, multiphoton pairs are passed through the channel with a higher probability. For this scenario, they significantly contribute to the double-click events. We ascribe to each such event a random value of the corresponding qubit. This protocol is characterized by classical probabilities and it certainly diminishes the nonclassical correlations between site $\mathrm{A}$ and site $\mathrm{B}$. Consequently, the value of the Bell parameter decreases more rapidly with increasing squeezing parameter $\xi$ compared to the case of discarding the double-click events.

Another important issue that appears in the scenario of copropagation, is the fact that for weak-to-moderate turbulence channels the advantages of the atmospheric links are negligible. For weak-intensity sources, when the state is approximately equal to the Bell state $(12)$, this fact can be easily explained by the low ratio $\left\langle\Delta \eta^{2}\right\rangle /\langle\eta\rangle^{2}$. Indeed, in this case the equality in Eq. 29) is satisfied almost exactly. However, as we can see in Fig. 2(b), even the presence of multiphoton pairs does not improve the value of the Bell parameter.

\section{COUNTERPROPAGATION}

The scenario of counterpropagation, which is represented in Fig. 1 (b), corresponds to channels with completely uncorrelated transmittances, $\eta_{\mathrm{A}}$ and $\eta_{\mathrm{B}}$. The PDT in this case reads as

$$
\mathcal{P}\left(\eta_{\mathrm{A}}, \eta_{\mathrm{B}}\right)=\mathcal{P}_{\mathrm{A}}\left(\eta_{\mathrm{A}}\right) \mathcal{P}_{\mathrm{B}}\left(\eta_{\mathrm{B}}\right)
$$

where $\mathcal{P}_{\mathrm{A}}\left(\eta_{\mathrm{A}}\right)$ and $\mathcal{P}_{\mathrm{B}}\left(\eta_{\mathrm{B}}\right)$ are the single-mode PDT for the corresponding channels. As a particular case, this scenario describes the situation when only one mode is sent through a turbulent atmosphere while another one is operated near the source, such as experimentally studied in Ref. 12 . In this case

$$
\mathcal{P}_{\mathrm{B}}\left(\eta_{\mathrm{B}}\right)=\delta\left(\eta_{\mathrm{B}}-\eta_{\mathrm{b}}\right)
$$

where $\eta_{\mathrm{b}}$ is the deterministic transmission coefficient of channel B.

In the case of a weak-intensity source, which is effectively described by the Bell state (11), Eqs. 25), (26), and (24) yield

$$
\begin{aligned}
& p_{0}=\left(1-\left\langle\eta_{\mathrm{A}}\right\rangle\right)\left(1-\left\langle\eta_{\mathrm{B}}\right\rangle\right), \\
& p_{1}=\left\langle\eta_{\mathrm{A}}\right\rangle\left(1-\left\langle\eta_{\mathrm{B}}\right\rangle\right)+\left\langle\eta_{\mathrm{B}}\right\rangle\left(1-\left\langle\eta_{\mathrm{A}}\right\rangle\right), \\
& p_{\mathcal{B}}=\left\langle\eta_{\mathrm{A}}\right\rangle\left\langle\eta_{\mathrm{B}}\right\rangle .
\end{aligned}
$$

This means that the corresponding fading channel is completely equivalent to the deterministic-loss channel with transmittances $\eta_{\mathrm{a}(\mathrm{b})}=\left\langle\eta_{\mathrm{A}(\mathrm{B})}\right\rangle$. Hence, in this case fading does not result in any advantages.

Our study has shown that the atmospheric links for the considered channels give the same result as the related deterministic-loss channels even for strong-intensity sources, when contributions from multiphoton pairs are essential. Results of the corresponding calculations are presented in Fig. 3. It is also important to note that the effect of double-click events is visible only for strongintensity sources (for large values of the squeezing parameter $\xi$ ) with weak- to moderate-turbulence channels [cf. Fig 3 (b)]. In all other cases the amount of contributions of multi-photon pairs, stray light, and dark counts at the receiver is not enough to diminish the value of the Bell parameter essentially.

\section{POSTSELECTION PROCEDURE}

In cases where verifications of Bell-inequality violations are impossible, one can try to improve the situation with a certain postselection procedure, using the technique proposed in Ref. [45]. For this purpose one sends intense light pulses in each channel, before the series of nonclassical-light pulses. With these pulses one can test the channel and then postselect the events with transmittances, $\eta_{\mathrm{A}(\mathrm{B})} \geq \eta_{\mathrm{ps}}$, exceeding a certain postselection threshold, $\eta_{\mathrm{ps}}$ (see Fig. 4). In this case, the time $\tau$ between the test and the nonclassical pulses should be smaller then the timescale of atmospheric variations.

The theoretical analysis of this scheme is based on the corresponding reformulation of the PDT. The singlemode PDT after postselection, $\widetilde{\mathcal{P}}_{\mathrm{A}(\mathrm{B})}\left(\eta_{\mathrm{A}(\mathrm{B})}\right)$, is given by

$$
\widetilde{\mathcal{P}}_{\mathrm{A}(\mathrm{B})}\left(\eta_{\mathrm{A}(\mathrm{B})}\right)=\frac{1}{\bar{F}_{\mathrm{A}(\mathrm{B})}\left(\eta_{\mathrm{ps}}\right)} \mathcal{P}_{\mathrm{A}(\mathrm{B})}\left(\eta_{\mathrm{A}(\mathrm{B})}\right)
$$

for $\eta_{\mathrm{A}(\mathrm{B})} \in\left[\eta_{\mathrm{ps}}, 1\right]$ and 0 elsewhere. Here

$$
\bar{F}_{\mathrm{A}(\mathrm{B})}\left(\eta_{\mathrm{ps}}\right)=\int_{\eta_{\mathrm{ps}}}^{1} \mathrm{~d} \eta \mathcal{P}_{\mathrm{A}(\mathrm{B})}(\eta)
$$

is the exceedance (complementary cumulative probability distribution) of the PDT, which is the probability that the transmittance will exceed the value of $\eta_{\mathrm{ps}}$. The calculations for the postselection procedure are performed with the PDT given in Eq. (35). It follows that

$$
\bar{F}_{\mathrm{AB}}\left(\eta_{\mathrm{ps}}\right)=\bar{F}_{\mathrm{A}}\left(\eta_{\mathrm{ps}}\right) \bar{F}_{\mathrm{B}}\left(\eta_{\mathrm{ps}}\right)
$$


Squeezing $(\mathrm{dB})$

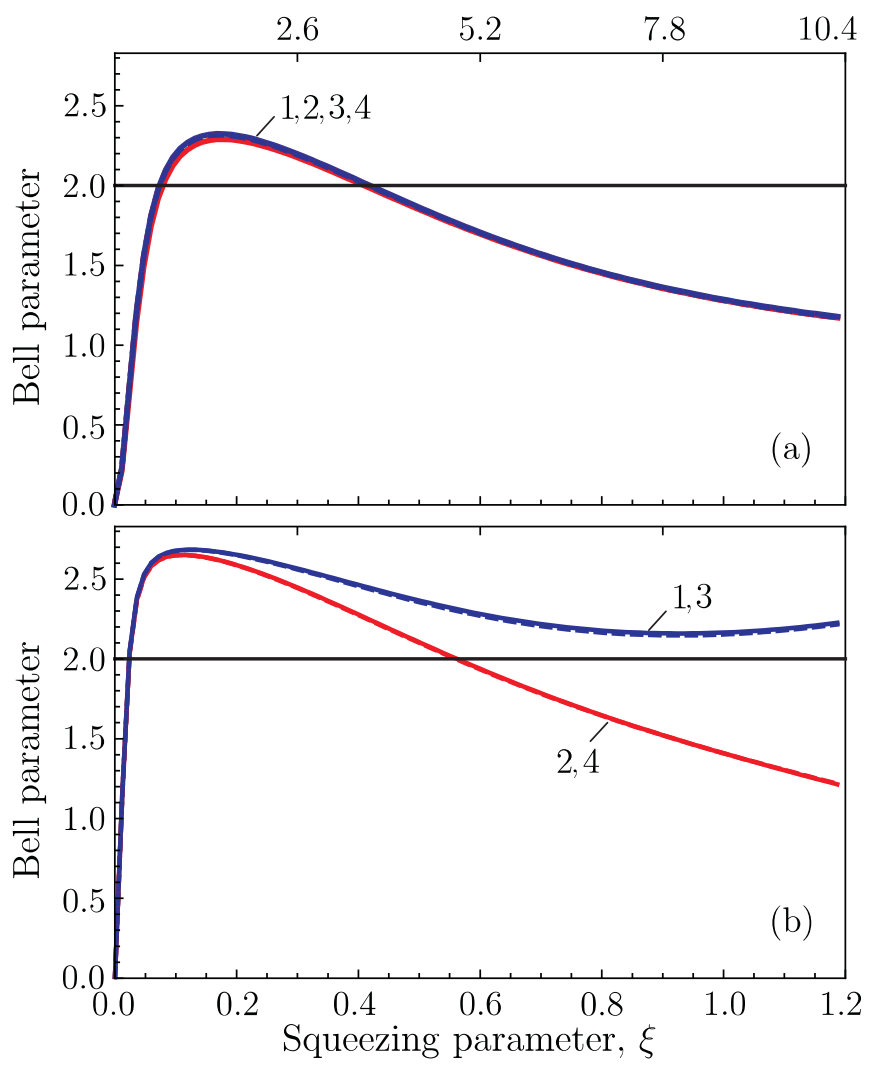

FIG. 3. (Color online) The Bell parameter, $\mathcal{B}$, vs the squeezing parameter, $\xi$, for the scenario of counterpropagation. The PDT is taken (a) for the strong-turbulence channel (cf. Appendix $(B)$, where the mean number of stray-light and dark counts is $\nu=1.7 \times 10^{-5}$, and (b) for the weak- to moderateturbulence channel (cf. Appendix A, with $\nu=3 \times 10^{-3}$. For both cases, lines 1 and 2 correspond to fading channels without and with the incorporation of double-clicks events, respectively. Lines 3 and 4 correspond to the deterministic-loss channels with $\eta_{a}=\eta_{b}=\left\langle\eta_{\mathrm{A}}\right\rangle=\left\langle\eta_{\mathrm{B}}\right\rangle$, without and with the incorporation of double-clicks events, respectively. The detection efficiency is $\eta_{c}=0.6$.

is the probability that the transmittances exceed the value of $\eta_{\mathrm{ps}}$ in both channels. This quantity characterizes the feasibility of the postselection procedure.

In Fig. 5 we represent the dependence of the Bell parameter on the postselection efficiency $\eta_{\mathrm{ps}}$. Under the considered conditions verification of the Bell-inequality violations is impossible without the postselection procedure due to the large dark-count and stray-light noise, characterized by the value of $\nu$. The postselection procedure certainly improves the situation.

\section{SUMMARY AND CONCLUSIONS}

To conclude, we note that the incorporation of doubleclick events and the absence of correlations in the trans-

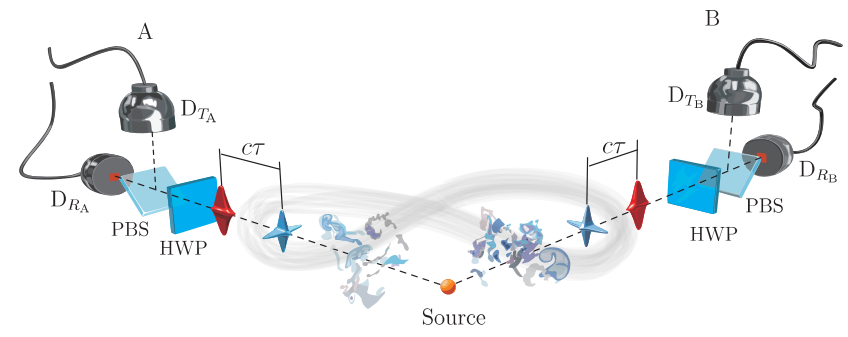

FIG. 4. (Color online) Bell-inequality test with the postselection procedure proposed in Ref. [45. Strong light pulses are sent in front of the nonclassical-light pulses in order to test the transmittance of the channels. The time $\tau$ is much less than the typical time of atmospheric variations. One considers only those events for which on both sides the measured transmittance $\eta_{\mathrm{A}(\mathrm{B})} \geq \eta_{\mathrm{ps}}$, where $\eta_{\mathrm{ps}}$ is the postselection threshold.

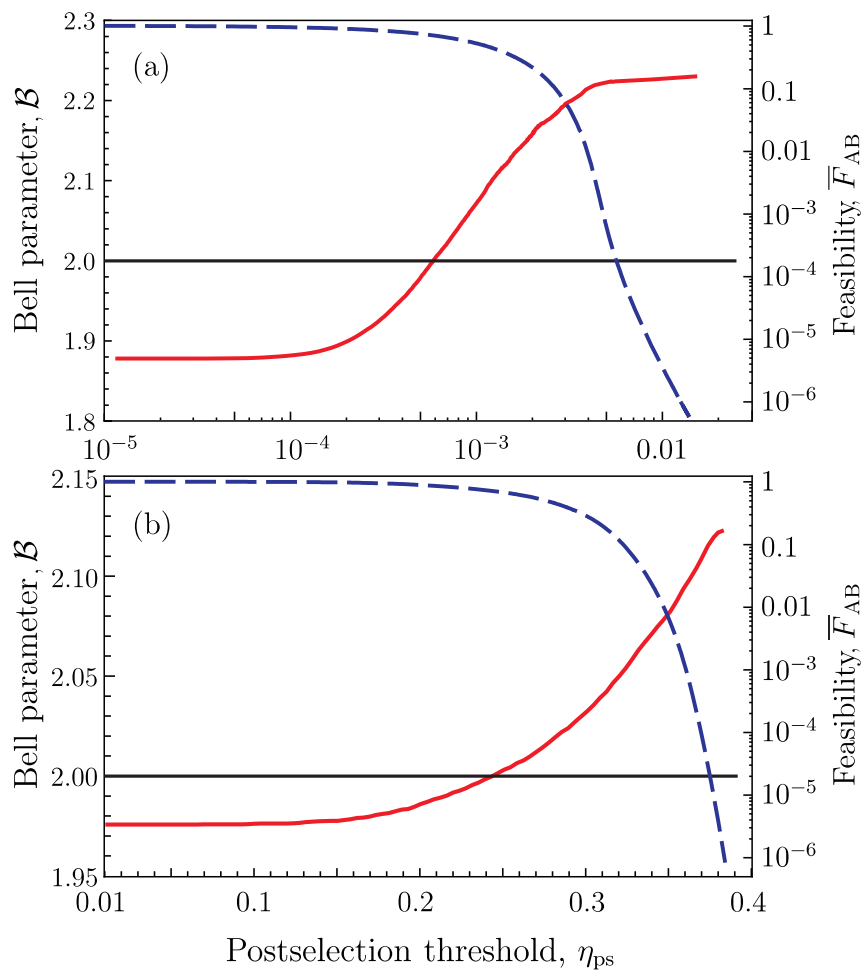

FIG. 5. (Color online) The Bell parameter, $\mathcal{B}$, vs the postselection efficiency, $\eta_{\mathrm{ps}}$, (solid line), and the feasibility [cf. Eq. [37)] of the postselection procedure, $\bar{F}_{\mathrm{AB}}$ (dashed line). Two scenarios are considered:(a) the strong-turbulence channel (cf. Appendix B), where the mean number of straylight and dark counts is $\nu=4 \times 10^{-4}$ and $\xi=0.25$, and (b) the weak- to moderate-turbulence channel (cf. Appendix A, where $\nu=2 \times 10^{-2}$ and the squeezing parameter is $\xi=0.31$. The detection efficiency is $\eta_{c}=0.6$.

mittances are certainly destructive factors for verifications of Bell-inequality violations in a turbulent atmosphere. In this paper we have theoretically studied the corresponding experiment with two types of channels: a 1.6-km channel with weak- to moderate-turbulent condi- 
tions and a 144-km channel with strong turbulence. We have found that the incorporation of double-click events does not destroy the advantages of fading channels in the scenario of copropagation. However, in the case of counterpropagation, with uncorrelated channels, the advantages cannot be utilized without additional procedures.

Resources of atmospheric turbulence in the counterpropagation scenario can be used with an additional postselection procedure studied above. This procedure appears to be feasible for both types of considered channels, i.e., for weak- to moderate and strong turbulence. We believe that our results will be useful for study of quantum communication through atmospheric channels.

\section{ACKNOWLEDGMENTS}

A.A.S., D.V., and W.V. acknowledge support from the Deutsche Forschungsgemeinschaft through Project No VO 501/21-1.

\section{Appendix A: Weak- to moderate-turbulence channel}

In this Appendix we remind the reader of the main result in Ref. 40, for the PDT in the elliptic-beam approximation with parameters appropriate for the weakto moderate-turbulence channel. In this case, the PDT is given by

$$
\mathcal{P}(\eta)=\frac{2}{\pi} \int_{\mathbb{R}^{4}} \mathrm{~d}^{4} \mathbf{v} \int_{0}^{\pi / 2} \mathrm{~d} \chi \rho_{G}(\mathbf{v} ; \boldsymbol{\mu}, \Sigma) \delta\left[\eta-\eta_{\mathrm{m}} \eta(\mathbf{v}, \chi)\right]
$$

Here $\mathbf{v}=\left(x_{0} y_{0} \Theta_{1} \Theta_{2}\right)^{\mathrm{T}}$ is a random vector, where $x_{0}$, $y_{0}$ are the beam-centroid coordinates, $\Theta_{1}, \Theta_{2}$ are parameters characterizing the elliptic deformations of the beam, $\rho_{G}(\mathbf{v} ; \boldsymbol{\mu}, \Sigma)$ is the Gaussian probability density of vector $\mathbf{v}$ with mean $\boldsymbol{\mu}$ and covariance matrix $\Sigma, \chi$ is a uniformly distributed angle characterizing the direction of the beam-spot ellipse, and $\eta_{\mathrm{m}}$ is the efficiency related to the deterministic losses that occur in the channel. The transmittance $\eta(\mathbf{v}, \chi)$ as a function of these parameters reads as

$$
\begin{aligned}
& \eta(\mathbf{v}, \chi)=\eta_{0}\left(\Theta_{1}, \Theta_{2}\right) \\
& \times \exp \left\{-\left[\frac{r_{0} / a}{R\left(\frac{2}{W_{\text {eff }}\left(\Theta_{1}, \Theta_{2}, \chi\right)}\right)}\right]^{\lambda\left(\frac{2}{W_{\text {eff }}\left(\Theta_{1}, \Theta_{2}, \chi\right)}\right)}\right\} .
\end{aligned}
$$

In this equation $r_{0}=\sqrt{x_{0}^{2}+y_{0}^{2}}$ is the distance between the beam and the aperture centers, $a$ is the radius of the receiver aperture,

$$
\begin{aligned}
& W_{\text {eff }}^{2}\left(\Theta_{1}, \Theta_{2}, \chi\right)=4 a^{2}\left[\mathcal { W } \left(\frac{4 a^{2}}{W_{1}\left(\Theta_{1}\right) W_{2}\left(\Theta_{2}\right)}\right.\right. \\
& \left.\left.\times e^{\frac{a^{2}}{W_{1}^{2}\left(\Theta_{1}\right)}\left\{1+2 \cos ^{2} \chi\right\}} e^{\frac{a^{2}}{W_{2}^{2}\left(\Theta_{2}\right)}\left\{1+2 \sin ^{2} \chi\right\}}\right)\right]^{-1}
\end{aligned}
$$

$\eta_{0}\left(\Theta_{1}, \Theta_{2}\right)$

$$
\begin{aligned}
& =1-\mathrm{I}_{0}\left(a^{2}\left[\frac{1}{W_{1}^{2}\left(\Theta_{1}\right)}-\frac{1}{W_{2}^{2}\left(\Theta_{2}\right)}\right]\right) e^{-a^{2}\left[\frac{1}{W_{1}^{2}\left(\Theta_{1}\right)}+\frac{1}{W_{2}^{2}\left(\Theta_{2}\right)}\right]} \\
& -2\left[1-e^{\left.-\frac{a^{2}}{2}\left(\frac{1}{W_{1}\left(\Theta_{1}\right)}-\frac{1}{W_{2}\left(\Theta_{2}\right)}\right)^{2}\right]}\right. \\
& \times \exp \left\{-\left[\frac{\frac{\left(W_{1}\left(\Theta_{1}\right)+W_{2}\left(\Theta_{2}\right)\right)^{2}}{\left|W_{1}^{2}\left(\Theta_{1}\right)-W_{2}^{2}\left(\Theta_{2}\right)\right|}}{R\left(\frac{1}{W_{1}\left(\Theta_{1}\right)}-\frac{1}{W_{2}\left(\Theta_{2}\right)}\right)}\right]^{\lambda\left(\frac{1}{W_{1}\left(\Theta_{1}\right)}-\frac{1}{W_{2}\left(\Theta_{2}\right)}\right)}\right\},
\end{aligned}
$$

$R(\xi)$ and $\lambda(\xi)$ are scale and shape functions, respectively,

$$
\begin{gathered}
R(\xi)=\left[\ln \left(2 \frac{1-\exp \left[-\frac{1}{2} a^{2} \xi^{2}\right]}{1-\exp \left[-a^{2} \xi^{2}\right] \mathrm{I}_{0}\left(a^{2} \xi^{2}\right)}\right)\right]^{-\frac{1}{\lambda(\xi)}}, \\
\lambda(\xi)=2 a^{2} \xi^{2} \frac{e^{-a^{2} \xi^{2}} \mathrm{I}_{1}\left(a^{2} \xi^{2}\right)}{1-\exp \left[-a^{2} \xi^{2}\right] \mathrm{I}_{0}\left(a^{2} \xi^{2}\right)} \\
\times\left[\ln \left(2 \frac{1-\exp \left[-\frac{1}{2} a^{2} \xi^{2}\right]}{1-\exp \left[-a^{2} \xi^{2}\right] \mathrm{I}_{0}\left(a^{2} \xi^{2}\right)}\right)\right]^{-1} \\
W_{i}^{2}=W_{0}^{2} \exp \Theta_{i}, \quad i=1,2,
\end{gathered}
$$

$W_{0}$ is the beam-spot radius at the source, $I_{i}(\xi)$ is the modified Bessel function of the $i$-th order, and $\mathcal{W}(\xi)$ is the Lambert $W$ function [47.

The elements of the covariance matrix $\Sigma$ and the vector of mean values $\boldsymbol{\mu}$ can be written in terms of the field correlation functions of the second and fourth orders. For conditions of weak to moderate turbulence when the Kolmogorov turbulence spectrum is applicable, the corresponding non-zero elements are given by

$$
\begin{gathered}
\left\langle\Theta_{1 / 2}\right\rangle=\ln \left[\frac{\left(1+2.96 \sigma_{R}^{2} \Omega^{\frac{5}{6}}\right)^{2}}{\Omega^{2} \sqrt{\left(1+2.96 \sigma_{R}^{2} \Omega^{\frac{5}{6}}\right)^{2}+1.2 \sigma_{R}^{2} \Omega^{\frac{5}{6}}}}\right] \\
\left\langle\Delta x_{0}^{2}\right\rangle=\left\langle\Delta y_{0}^{2}\right\rangle=0.33 W_{0}^{2} \sigma_{R}^{2} \Omega^{-\frac{7}{6}} \\
\left\langle\Delta \Theta_{1 / 2}^{2}\right\rangle=\ln \left[1+\frac{1.2 \sigma_{R}^{2} \Omega^{\frac{5}{6}}}{\left(1+2.96 \sigma_{R}^{2} \Omega^{\frac{5}{6}}\right)^{2}}\right]
\end{gathered}
$$




$$
\left\langle\Delta \Theta_{1} \Delta \Theta_{2}\right\rangle=\ln \left[1-\frac{0.8 \sigma_{R}^{2} \Omega^{\frac{5}{6}}}{\left(1+2.96 \sigma_{R}^{2} \Omega^{\frac{5}{6}}\right)^{2}}\right]
$$

where the Rytov parameter $\sigma_{R}^{2}$ is defined in Eq. 14, $\Omega=\frac{k W_{0}^{2}}{2 L}$ is the Fresnel parameter, $k$ is the wave number, and $L$ is the propagation distance. For the considered 1.6-km channel (cf. Ref. [46]), the corresponding parameters are $\sigma_{R}^{2}=1.5, \Omega=0.98, W_{0}=0.02[\mathrm{~m}], a=0.04[\mathrm{~m}]$, and $L=1.6[\mathrm{~km}]$. The corresponding elements of the covariance matrix $\Sigma$ and the vector of mean values $\boldsymbol{\mu}$ can be calculated explicitly,

$$
\begin{gathered}
\Sigma=\left(\begin{array}{cccc}
2 \times 10^{-4}\left[\mathrm{~m}^{2}\right] & 0 & 0 & 0 \\
0 & 2 \times 10^{-4}\left[\mathrm{~m}^{2}\right] & 0 & 0 \\
0 & 0 & 0.06 & -0.04 \\
0 & 0 & -0.04 & 0.06
\end{array}\right), \\
\boldsymbol{\mu}=\left(\begin{array}{llll}
0 & 0 & 1.69 & 1.69
\end{array}\right)^{\mathrm{T}},
\end{gathered}
$$

which makes it possible to perform the integration in Eq. A1 numerically.

The numerical integration can be performed within the Monte Carlo method. In this paper we have to calculate the means of a certain function of transmittance, $\langle f(\eta)\rangle$. For this purpose we should simulate the $N$ values of the vector $\mathbf{v}$ and the angle $\chi$. The needed quantity is then estimated as

$$
\langle f(\eta)\rangle=\frac{1}{N} \sum_{i=1}^{N} f\left(\eta_{\mathrm{m}} \eta\left(\mathbf{v}_{i}, \chi_{i}\right)\right),
$$

where $\eta\left(\mathbf{v}_{i}, \chi_{i}\right)$ is obtained from Eq. $\mathrm{A} 2$. The deterministic losses associated with absorption and scattering by the atmosphere and optical elements correspond to the efficiency $\eta_{\mathrm{m}}=0.75$.

\section{Appendix B: Strong-turbulence channel}

In this Appendix we consider how to estimate the PDT from the experimental data in 45 for the $144 \mathrm{~km}$ channel on the Canary Islands. The Rytov parameter for this channel satisfies the condition $\sigma_{R}^{2} \gg 1$, and therefore the optical turbulence in the channel is strong. As shown in [40, for strong-turbulence conditions one can also use the elliptic-beam approximation considered in Appendix A However, in this case Eqs. A8, A9, A10, and A11 are not valid.

It has been demonstrated both theoretically (cf. Ref. [40]) and experimentally (cf. Ref. [45]) that for such conditions the PDT can be approximated by the truncated log-normal distribution,

$$
\mathcal{P}(\eta)=\frac{1}{\eta F\left(\eta_{\mathrm{m}}\right) \sigma \sqrt{2 \pi}} \exp \left[-\frac{(\ln \eta+\mu)^{2}}{2 \sigma^{2}}\right]
$$

for $\eta \in\left[0, \eta_{\mathrm{m}}\right]$ and 0 elsewhere, where $\sigma$ and $\mu$ are parameters of this distribution, and $F\left(\eta_{\mathrm{m}}\right)$ is the cumulative probability distribution of the (nontruncated) log-normal distribution at point $\eta=\eta_{\mathrm{m}}$, which corresponds to deterministic losses in the channel.

From Ref. 45] we already know important parameters: the mean radiation energy (in $\hbar \omega$ units), $\langle q\rangle=234$, in the counting time interval, its standard deviation $\left\langle\Delta q^{2}\right\rangle=349^{2}$, and the mean losses $\langle\eta\rangle=10^{-3}$. By using the relation

$$
\frac{\left\langle\Delta \eta^{2}\right\rangle}{\langle\eta\rangle^{2}}=\frac{\left\langle\Delta q^{2}\right\rangle}{\langle q\rangle^{2}}
$$

one gets $\left\langle\Delta \eta^{2}\right\rangle=2.2 \times 10^{-6}$. The parameters $\sigma$ and $\mu$ can now be approximately evaluated as

$$
\begin{gathered}
\sigma^{2} \approx \ln \left(1+\frac{\left\langle\Delta \eta^{2}\right\rangle}{\langle\eta\rangle^{2}}\right), \\
\mu \approx-\ln \left(\frac{\langle\eta\rangle}{\sqrt{1+\frac{\left\langle\Delta \eta^{2}\right\rangle}{\langle\eta\rangle^{2}}}}\right) .
\end{gathered}
$$

For the considered channel these parameters are $\sigma=1.08$, $\mu=7.49$. The deterministic losses, $0.1 \mathrm{~dB} / \mathrm{km}$ (cf. Ref. 48, ) as well as the losses related to the optical elements correspond to the efficiency $\eta_{\mathrm{m}}=0.04$.

\section{Appendix C: Photocounting probabilities for the PDC source}

In this Appendix we discuss the probabilities $P_{\text {same }}\left(\theta_{\mathrm{A}}, \theta_{\mathrm{B}}\right)$ and $P_{\text {different }}\left(\theta_{\mathrm{A}}, \theta_{\mathrm{B}}\right)$ for the case of a PDC source, cf. Eq. (17). For convenience we use the GaluberSudarshan $P$-representation $[2931$. The $P$ function of the PDC state, (9), cannot be represented in terms of regular functions. For this reason we use the corresponding characteristic function,

$$
\Phi(\boldsymbol{\beta})=\operatorname{Tr}\left[\exp \left(\boldsymbol{\beta} \hat{\boldsymbol{a}}^{\dagger}\right) \exp \left(-\boldsymbol{\beta}^{*} \hat{\boldsymbol{a}}\right) \hat{\rho}\right]
$$

where $\hat{\boldsymbol{a}}$ is the vector consisting of field-mode annihilation operators, $\boldsymbol{\beta}$ is the corresponding complex-number vector of the characteristic-function arguments, and $\hat{\rho}$ is the density operator.

In this representation Eq. (6) reads as

$$
\begin{aligned}
& \int_{i_{\mathrm{A}}, i_{\mathrm{B}}}\left(\theta_{\mathrm{A}}, \theta_{\mathrm{B}}\right)= \\
& \int_{-\infty}^{+\infty} d^{8} \boldsymbol{\beta} \Phi_{\text {out }}(\boldsymbol{\beta})\left[K_{C}\left(\beta_{i_{\mathrm{A}}}\right) K_{C}\left(\beta_{i_{\mathrm{B}}}\right) K_{0}\left(\beta_{j_{\mathrm{A}}}\right) K_{0}\left(\beta_{j_{\mathrm{B}}}\right)\right. \\
& +K_{C}\left(\beta_{i_{\mathrm{A}}}\right) K_{C}\left(\beta_{i_{\mathrm{B}}}\right) K_{0}\left(\beta_{j_{\mathrm{A}}}\right) K_{C}\left(\beta_{j_{\mathrm{B}}}\right) \\
& +K_{C}\left(\beta_{i_{\mathrm{A}}}\right) K_{C}\left(\beta_{i_{\mathrm{B}}}\right) K_{C}\left(\beta_{j_{\mathrm{A}}}\right) K_{0}\left(\beta_{j_{\mathrm{B}}}\right) \\
& \left.+K_{C}\left(\beta_{i_{\mathrm{A}}}\right) K_{C}\left(\beta_{i_{\mathrm{B}}}\right) K_{C}\left(\beta_{j_{\mathrm{A}}}\right) K_{C}\left(\beta_{j_{\mathrm{B}}}\right)\right] .
\end{aligned}
$$


Here $\Phi_{\text {out }}(\boldsymbol{\beta})$ is the characteristic function of the state after passing the atmosphere, $d^{8} \boldsymbol{\beta}=$ $d^{2} \beta_{T_{\mathrm{A}}} d^{2} \beta_{R_{\mathrm{A}}} d^{2} \beta_{T_{\mathrm{B}}} d^{2} \beta_{R_{\mathrm{B}}}$,

$$
\begin{aligned}
& K_{0}(\beta)=\frac{1}{\pi \eta_{c}} \exp \left[-\frac{|\beta|^{2}}{\eta_{c}}-\nu\right], \\
& K_{C}(\beta)=\delta(\beta)-K_{0}(\beta) .
\end{aligned}
$$

For more details on the notations, see the explanations following Eq. (8).

The output modes of the polarization analyzers, $\boldsymbol{\beta}=\left\{\beta_{T_{\mathrm{A}}}, \beta_{R_{\mathrm{A}}}, \beta_{T_{\mathrm{B}}}, \beta_{R_{\mathrm{B}}}\right\}$, are related to the corresponding input modes, $\boldsymbol{\beta}=\left\{\beta_{\mathrm{H}_{\mathrm{A}}}, \beta_{\mathrm{V}_{\mathrm{A}}}, \beta_{\mathrm{H}_{\mathrm{B}}}, \beta_{\mathrm{V}_{\mathrm{B}}}\right\}$, via the inputoutput relations,

$$
\begin{aligned}
& \beta_{\mathrm{H}_{\mathrm{A}(\mathrm{B})}}=\beta_{T_{\mathrm{A}(\mathrm{B})}} \cos \theta_{\mathrm{A}(\mathrm{B})}-\beta_{R_{A(\mathrm{~B})}} \sin \theta_{\mathrm{A}(\mathrm{B})}, \\
& \beta_{\mathrm{V}_{\mathrm{A}(\mathrm{B})}}=\beta_{T_{\mathrm{A}(\mathrm{B})}} \sin \theta_{\mathrm{A}(\mathrm{B})}+\beta_{R_{\mathrm{A}(\mathrm{B})}} \cos \theta_{\mathrm{A}(\mathrm{B})}
\end{aligned}
$$

[cf. also the corresponding operator form given by Eqs. [15) and [16)]. Here the indexes $\mathrm{H}_{\mathrm{A}(\mathrm{B})}$ and $\mathrm{V}_{\mathrm{A}(\mathrm{B})}$ denote the corresponding horizontal and vertical polarization modes, respectively. The characteristic function of the light passed through the receiver apertures, $\Phi_{\text {out }}\left(\beta_{\mathrm{H}_{\mathrm{A}}}, \beta_{\mathrm{V}_{\mathrm{A}}}, \beta_{\mathrm{H}_{\mathrm{B}}}, \beta_{\mathrm{V}_{\mathrm{B}}}\right)$, is expressed in terms of the characteristic function of the light generated by the source, $\Phi_{\text {in }}\left(\beta_{\mathrm{H}_{\mathrm{A}}}, \beta_{\mathrm{V}_{\mathrm{A}}}, \beta_{\mathrm{H}_{\mathrm{B}}}, \beta_{\mathrm{V}_{\mathrm{B}}}\right)$, according to the quantum-state input-output relation for fading channels (cf. Refs. [14, 15]),

$$
\begin{aligned}
& \Phi_{\text {out }}\left(\beta_{\mathrm{H}_{\mathrm{A}}}, \beta_{\mathrm{V}_{\mathrm{A}}}, \beta_{\mathrm{H}_{\mathrm{B}}}, \beta_{\mathrm{V}_{\mathrm{B}}}\right) \\
& =\left\langle\Phi_{\text {in }}\left(\sqrt{\eta_{\mathrm{A}}} \beta_{\mathrm{H}_{\mathrm{A}}}, \sqrt{\eta_{\mathrm{A}}} \beta_{\mathrm{V}_{\mathrm{A}}}, \sqrt{\eta_{\mathrm{B}}} \beta_{\mathrm{H}_{\mathrm{B}}}, \sqrt{\eta_{\mathrm{B}}} \beta_{\mathrm{V}_{\mathrm{B}}}\right)\right\rangle,
\end{aligned}
$$

where the averaging with the channel transmissions is defined by Eq. 22.

The characteristic function of the PDC state, cf. Eq. (9), is given by

$$
\begin{aligned}
\Phi_{\mathrm{in}}\left(\beta_{\mathrm{H}_{\mathrm{A}}}, \beta_{\mathrm{V}_{\mathrm{A}}}, \beta_{\mathrm{H}_{\mathrm{B}}}, \beta_{\mathrm{V}_{\mathrm{B}}}\right) & =\exp \left[-\frac{\tanh ^{2} \xi\left|\beta_{\mathrm{V}_{\mathrm{A}}}\right|^{2}+\tanh ^{2} \xi\left|\beta_{\mathrm{H}_{\mathrm{B}}}\right|^{2}-\tanh \xi\left(\beta_{\mathrm{V}_{\mathrm{A}}} \beta_{\mathrm{H}_{\mathrm{B}}}+\beta_{\mathrm{V}_{\mathrm{A}}}^{*} \beta_{\mathrm{H}_{\mathrm{B}}}^{*}\right)}{1-\tanh ^{2} \xi}\right] \\
& \times \exp \left[-\frac{\tanh ^{2} \xi\left|\beta_{\mathrm{H}_{\mathrm{A}}}\right|^{2}+\tanh ^{2} \xi\left|\beta_{\mathrm{V}_{\mathrm{B}}}\right|^{2}+\tanh \xi\left(\beta_{\mathrm{H}_{\mathrm{A}}} \beta_{\mathrm{V}_{\mathrm{B}}}+\beta_{\mathrm{H}_{\mathrm{A}}}^{*} \beta_{\mathrm{V}_{\mathrm{B}}}^{*}\right)}{1-\tanh ^{2} \xi}\right] .
\end{aligned}
$$

Substituting this expression into Eq. C7 and then utilizing it in Eq. (C2) together with Eqs. (C5) and (C6), one gets the explicit form for $P_{i_{\mathrm{A}}, i_{\mathrm{B}}}\left(\theta_{\mathrm{A}}, \theta_{\mathrm{B}}\right)$. Finally the result used in Eqs. (1) and (2) which leads to the expressions for $P_{\text {same }}\left(\theta_{\mathrm{A}}, \theta_{\mathrm{B}}\right)$ and $P_{\text {different }}\left(\theta_{\mathrm{A}}, \theta_{\mathrm{B}}\right)$ is given by Eq. (17).

\section{Appendix D: Bell states}

In this Appendix we discuss the derivation of the expression for the maximal value of the Bell parameter [cf. Eq. [23] ] in the case of weak-intensity sources described by the Bell state $\sqrt{11}$ and $\sqrt{12}$. First, we recall the explicit form for the density operator of the corresponding state after passing through the atmosphere (cf. Ref. [18]),

$\hat{\rho}=p_{0} \hat{\rho}_{0}+p_{\mathrm{H}_{\mathrm{A}}} \hat{\rho}_{\mathrm{H}_{\mathrm{A}}}+p_{\mathrm{V}_{\mathrm{A}}} \hat{\rho}_{\mathrm{V}_{\mathrm{A}}}+p_{\mathrm{H}_{\mathrm{B}}} \hat{\rho}_{\mathrm{H}_{\mathrm{B}}}+p_{\mathrm{V}_{\mathrm{B}}} \hat{\rho}_{\mathrm{V}_{\mathrm{B}}}+p_{\mathcal{B}} \hat{\rho}_{\mathcal{B}}$,

(D1)

where $\hat{\rho}_{0}$ is the density operator of the vacuum state, $\hat{\rho}_{\mathrm{H}_{\mathrm{A}(\mathrm{B})}}, \hat{\rho}_{\mathrm{V}_{\mathrm{A}(\mathrm{B})}}$ are the single-photon density operators in the corresponding mode, and $\hat{\rho}_{\mathcal{B}}=|\mathcal{B}\rangle\langle\mathcal{B}|$ is the density operator of the Bell state [cf. Eq. [11)]. Here $p_{0}$ is the probability that no photons from the source will reach the receivers [cf. Eq. [25]], $p_{\mathcal{B}}$ is the probability that both photons of the Bell state will reach the receivers [cf. Eq. 24],

$$
p_{\mathrm{H}_{\mathrm{A}(\mathrm{B})}}=p_{\mathrm{v}_{\mathrm{A}(\mathrm{B})}}=\frac{1}{2}\left\langle\eta_{\mathrm{A}(\mathrm{B})}\left(1-\eta_{\mathrm{B}(\mathrm{A})}\right)\right\rangle
$$

is the probability that only one photon in the corresponding mode reaches the receivers such that

$$
p_{1}=p_{\mathrm{H}_{\mathrm{A}}}+p_{\mathrm{H}_{\mathrm{B}}}+p_{\mathrm{v}_{\mathrm{A}}}+p_{\mathrm{v}_{\mathrm{B}}}
$$

is the probability that only one photon will reaches the receivers [cf. Eq. 26)].

Next we utilize the operator form of the polarizationanalyzer input-output relations [cf. Eqs. (15) and (16)] in Eqs. (7) and (8). The result together with the density operator, (D1), is substituted in Eq. (6), which gives us the probabilities $P_{i_{\mathrm{A}}, i_{\mathrm{B}}}\left(\theta_{\mathrm{A}}, \theta_{\mathrm{B}}\right)$. By using these probabilities in Eqs. (1), (2), and (3) one gets the correlation coefficient,

$$
\begin{aligned}
& E\left(\theta_{\mathrm{A}}, \theta_{\mathrm{B}}\right)= \\
& -\frac{p_{\mathcal{B}} \eta_{c}^{2} e^{2 \nu} \cos \left[2\left(\theta_{\mathrm{A}}-\theta_{\mathrm{B}}\right)\right]}{p_{\mathcal{B}}\left(e^{2 \nu}+\eta_{c}-1\right)^{2}+p_{0}\left(e^{2 \nu}-1\right)^{2}+p_{1}\left(e^{2 \nu}-1\right)\left(e^{2 \nu}+\eta_{c}-1\right)} .
\end{aligned}
$$

For the particular values of the polarization angles, $\left(\theta_{\mathrm{A}}^{(1)}, \theta_{\mathrm{B}}^{(1)}, \theta_{\mathrm{A}}^{(2)}, \theta_{\mathrm{B}}^{(2)}\right)=\left(0, \frac{\pi}{8}, \frac{\pi}{4}, \frac{3 \pi}{8}\right)$, the Bell parameter $\mathcal{B}$, cf. Eq. (4), takes the value given by Eq. (23). 


\section{Appendix E: Bell-inequality test with discarding double-click events}

In this Appendix we remind the reader of some results in Ref. [18, where the effect of double-click events has not been included. In this case the last three terms in Eq. (6) vanish. For the PDC source the probabilities $P_{\text {same }}\left(\theta_{\mathrm{A}}, \theta_{\mathrm{B}}\right)$ and $P_{\text {different }}\left(\theta_{\mathrm{A}}, \theta_{\mathrm{B}}\right)$, read as

$$
\begin{aligned}
& P_{\mathrm{i}}\left(\theta_{\mathrm{A}}, \theta_{\mathrm{B}}\right)= \\
& 2\left(1-\tanh ^{2} \xi\right)^{4}\left[\left\langle\frac{\exp (-2 \nu)}{C_{0}+C_{1 \mathrm{~A}}+C_{1 \mathrm{~B}}+C_{\mathrm{i}}}\right\rangle-\left\langle\frac{\exp (-3 \nu)}{C_{0}+C_{1 \mathrm{~A}}}\right\rangle-\left\langle\frac{\exp (-3 \nu)}{C_{0}+C_{1 \mathrm{~B}}}\right\rangle+\left\langle\frac{\exp (-4 \nu)}{C_{0}}\right\rangle\right],
\end{aligned}
$$

where $i=\{$ same, different $\}$, for coefficients see Eqs. 18)-(21). For the weak-intensity source, described by the Bell state [cf. Eq [11]], the maximal value of the Bell parameter is given by

$$
\mathcal{B}=\frac{2 \sqrt{2} p_{\mathcal{B}} \eta_{c}^{2} e^{2 \nu}}{p_{\mathcal{B}}\left[\left(1-\eta_{c}\right)\left(e^{\nu}-2\right)+e^{\nu}\right]^{2}+2 p_{1}\left[e^{\nu}-1\right]\left[\eta_{c} e^{\nu}+2\left(e^{\nu}-1\right)\left(1-\eta_{c}\right)\right]+4 p_{0}\left[e^{\nu}-1\right]^{2}},
$$

for more details see the explanations following Eq. 23 .

[1] H. Takesue, S. W. Nam, Q. Zhang, R. H. Hadfield, T. Honjo, K. Tamaki, and Y. Yamamoto, Quantum Key Distribution over $40 \mathrm{~dB}$ Channel Loss Using Superconducting Single Photon Detectors, Nature Photon. 1, 343 (2007).

[2] S. Nauerth, F. Moll, M. Rau, C. Fuchs, J. Horwath, S. Frick, and H. Weinfurter, Air-to-Ground Quantum Communication, Nat. Photon. 7, 382 (2013).

[3] J.-Yu Wang et al., Direct and Full-Scale Experimental Verifications Towards Ground-Satellite Quantum Key Distribution, Nat. Photon. 7, 387 (2013).

[4] G. Vallone, D. Bacco, D. Dequal, S. Gaiarin, V. Luceri, G. Bianco, and P. Villoresi, Experimental Satellite Quantum Communications, Phys. Rev. Lett. 115, 040502 (2015).

[5] D. Dequal, G. Vallone, D. Bacco, S. Gaiarin, V. Luceri, G. Bianco, and P. Villoresi, Experimental Single Photon Exchange along a Space Link of 7000 km, Phys. Rev. A 93, 010301 (2016).

[6] G. Vallone, D. Dequal, M. Tomasin, F. Vedovato, M. Schiavon, V. Luceri, G. Bianco, and P. Villoresi, Quantum interference along satellite-ground channels, Phys. Rev. Lett. 116, 253601 (2016).

[7] J.-P. Bourgoin et al., A Comprehensive Design and Performance Analysis of Low Earth Orbit Satellite Quantum Communication, New J. Phys. 15, 023006 (2013).

[8] N. Brunner, D. Cavalcanti, S. Pironio, V. Scarani, and S. Wehner, Bell Nonlocality, Rev. Mod. Phys. 86, 419 (2014).

[9] A. K. Ekert, Quantum Cryptography Based on Bell's Theorem, Phys. Rev. Lett. 67, 661 (1991).

[10] A. Acín, N. Gisin, and L. Masanes, From Bells Theorem to Secure Quantum Key Distribution, Phys. Rev. Lett. 97, 120405 (2006).
[11] A. Acín, N. Brunner, N. Gisin, S. Massar, S. Pironio, and V. Scarani, Device-Independent Security of Quantum Cryptography against Collective Attacks, Phys. Rev. Lett. 98, 230501 (2007).

[12] R. Ursin et al., Entanglement-Based Quantum Communication over $144 \mathrm{~km}$, Nature Phys. 3, 481 (2007).

[13] A. Fedrizzi, R. Ursin, T. Herbst, M. Nespoli, R. Prevedel, T. Scheidl, F. Tiefenbacher, T. Jennewein, and A. Zeilinger, High-Fidelity Transmission of Entanglement over a High-Loss Free-Space Channel, Nature Phys. 5, 389 (2009).

[14] A. A. Semenov and W. Vogel, Quantum Light in the Turbulent Atmosphere, Phys. Rev. A 80, 021802(R) (2009).

[15] D. Yu. Vasylyev, A. A. Semenov and W. Vogel, Toward Global Quantum Communication: Beam Wandering Preserves Nonclassicality, Phys. Rev. Lett. 108, 220501 (2012).

[16] H. Avetisyan and C. H. Monken, Higher order correlation beams in atmosphere under strong turbulence conditions, Opt. Express 24, 2318 (2016).

[17] Y. Zhang, Sh. Prabhakar, A. H. Ibrahim, F. S. Roux, A. Forbes, and Th. Konrad, Experimentally observed decay of high-dimensional entanglement through turbulence, Phys. Rev. A 94, 032310 (2016).

[18] A. A. Semenov and W. Vogel, Entanglement Transfer through the Turbulent Atmosphere, Phys. Rev. A 81, 023835 (2010); 85, 019908(E) (2012).

[19] X. Ma, C. H. F. Fung, and H. K. Lo, Quantum Key Distribution with Entangled Photon Sources, Phys. Rev. A 76, 012307 (2007).

[20] P. Kok and S. L. Braunstein, Postselected Versus Nonpostselected Quantum Teleportation Using Parametric Down-Conversion, Phys. Rev. A 61, 042304 (2000). 
[21] A. A. Semenov and W. Vogel, Fake Violations of the Quantum Bell-Parameter Bound, Phys. Rev. A 83, 032119 (2011); 85, 049904(E) (2012).

[22] N. J. Beaudry, T. Moroder, and N. Lütkenhaus, Squashing Models for Optical Measurements in Quantum Communication, Phys. Rev. Lett. 101, 093601 (2008).

[23] T. Moroder, O. Gühne, N. Beaudry, M. Piani, and N. Lütkenhaus, Entanglement Verification with Realistic Measurement Devices via Squashing Operations, Phys. Rev. A 81, 052342 (2010).

[24] C. H. F. Fung, H. F. Chau, and H.-K. Lo, Universal Squash Model for Optical Communications Using Linear Optics and Threshold Detectors, Phys. Rev. A 84, 020303(R) (2011).

[25] J. F. Clauser, M. A. Horn, A. Shimony, and R. A. Holt, Proposed Experiment to Test Local Hidden Variable Theories, Phys. Rev. Lett. 23, 880 (1969).

[26] L. Mandel and E. Wolf, Optical Coherence and Quantum Optics (Cambridge University Press, Cambridge, 1995).

[27] P. L. Kelley and W. H. Kleiner, Theory of Electromagnetic Field Measurement and Photoelectron Counting, Phys. Rev. 136, A316 (1964).

[28] A. A. Semenov, A. V. Turchin and H. V. Gomonay, Detection of Quantum Light in the Presence of Noise, Phys. Rev. A 78, 055803 (2008); 79, 019902(E) (2009).

[29] R. J. Glauber, Photon Correlations, Phys. Rev. Lett. 10 84 (1963).

[30] R. J. Glauber, Coherent and Incoherent States of the Radiation Field, Phys. Rev. A 131, 2766 (1963).

[31] E. C. G. Sudarshan, Equivalence of Semiclassical and Quantum Mechanical Descriptions of Statistical Light Beams, Phys. Rev. Lett. 10, 277 (1963).

[32] V. Tatarskii, The Effect of the Turbulent Atmosphere on Wave Propagation (Israel Program for Scientific Translations, Jerusalem, 1971).

[33] A. Ishimaru, Wave Propagation and Scattering in Random Media (Academic Press, NY, 1978).

[34] L. Andrews, R. Phillips, and C. Hopen, Laser Beam Scintillation with Applications (SPIE Press, Washington, 2001).

[35] L. Andrews and R. Phillips, Laser Beam Propagation through Random Media (SPIE Press, Washington, 2005).

[36] R. L. Fante, Electromagnetic Beam Propagation in Turbulent Media, Proc. IEEE 63, 1669 (1975).
[37] R. L. Fante, Electromagnetic beam propagation in turbulent media: An update, Proc. IEEE 68, 1424 (1980).

[38] G. P. Berman and A. A. Chumak, Photon Distribution Function for Long-Distance Propagation of Partially Coherent Beams through the Turbulent Atmosphere, Phys. Rev. A 74, 013805 (2006).

[39] O. O. Chumak and R. A. Baskov, Strong Enhancing Effect of Correlations of Photon Trajectories on Laser Beam Scintillations, Phys. Rev. A 93, 033821 (2016).

[40] D. Vasylyev, A. A. Semenov, and W. Vogel, Atmospheric Quantum Channels with Weak and Strong Turbulence, Phys. Rev. Lett. 117, 090501 (2016).

[41] P. Diament and M. C. Teich, Photodetection of LowLevel Radiation through the Turbulent Atmosphere, J. Opt. Soc. Am. 60, 1489 (1970).

[42] J. Peřina, On the photon counting statistics of light passing through an inhomogeneous random medium, Czech. J. Phys. 22, 1075 (1972).

[43] J. Peřina, V. Peřinova, M. C. Teich, and P. Diament, Two Descriptions for the Photocounting Detection of Radiation Passed through a Random Medium: A Comparison for the Turbulent Atmosphere, Phys. Rev. A 7, 1732 (1973).

[44] P. Milonni, J. Carter, Ch. Peterson, and R. Hughes, Effects of Propagation through Atmospheric Turbulence on Photon Statistics, J. Opt. B 6, S742 (2004).

[45] I. Capraro, A. Tomaello, A. Dall'Arche, F. Gerlin, R. Ursin, G. Vallone, and P. Villoresi, Impact of Turbulence in Long Range Quantum and Classical Communications, Phys. Rev. Lett. 109, 200502 (2012).

[46] V. C. Usenko, B. Heim, C. Peuntinger, C. Wittmann, C. Marquardt, G. Leuchs, and R. Filip, Entanglement of Gaussian States and the Applicability to Quantum Key Distribution over Fading Channels, New J. Phys. 14, 093048 (2012).

[47] R. Corless, G. Gonnet, D. Hare, D. Jeffrey, and D. Knuth, On the Lambert W Function, Adv. Comput. Math. 5, 329 (1996).

[48] V. Scarani, H. Bechmann-Pasquinucci, N. J. Cerf, M. Dušek, N. Lütkenhaus, and M. Peev, The security of practical quantum key distribution, Rev. Mod. Phys. 81, 1301 (2009). 